\title{
Changes in the organization of surface antigens during in-vitro capacitation of boar spermatozoa as detected by monoclonal antibodies
}

\author{
N. Saxena, R. N. Peterson, S. Sharif, N. K. Saxena and L. D. Russell \\ Department of Physiology, School of Medicine, Southern Illinois University, Carbondale, \\ Illinois 62901, U.S.A.
}

\begin{abstract}
Summary. Monoclonal antibodies specific for three major plasma membrane (PM) proteins, previously referenced as PM protein $2 \cdot 0,4 \cdot 85$ and 5·0, and one specific for an unreferenced PM protein $\left(M_{\mathrm{r}} 80000\right)$ were used with indirect fluorescence microscopy to detect the effects of capacitation on the localization of these PM proteins. In ejaculated or cauda spermatozoa, incubation in the capacitating medium caused the appearance of fluorescence in the flagellum and either a loss of fluorescence on the PM overlying the sperm head (PM proteins of 5.0 and $M_{\mathrm{r}} 80000$ ) or a delocalization of fluorescence on the head PM (PM proteins 2.0 and 4.85 ). Labelling spermatozoa with divalent antibody and then capacitating them indicated the PM protein 5.0 and that of $M_{\mathrm{r}} 80000$ migrated out of the head plasma membrane into the flagellar PM during capacitation. These antigens re-entered the head PM when fresh seminal plasma was added after the capacitation period or when energy metabolism was inhibited by azide. Cytochalasin $\mathrm{D}$, an inhibitor of the polymerization of actin, prevented movement of PM protein 5.0 and that of $M_{\mathrm{r}} 80000$ of the head PM into the flagellum during incubation in the capacitation medium and prevented re-entry of these antigens from the flagellum into the head PM after incubation in this medium. Localization changes occurring with capacitation were time-dependent but independent of the method of preparing samples for microscopy. For the major PM proteins 4.85 and 5.0 , a much smaller percentage of caput spermatozoa $(\sim 20 \%)$ showed specific localization changes compared to those of the cauda $(\sim 80 \%)$. Chelation of $\mathrm{Ca}^{2+}$ inhibited these changes in ejaculated spermatozoa and fresh seminal plasma, added to capacitated spermatozoa, restored the localization pattern characteristic of uncapacitated spermatozoa. These observations suggest that the organization of major proteins in the plasma membrane overlying the sperm head is altered during capacitation. These changes are reversible, are dependent on sperm maturation and also appear to involve actin filament interactions with the plasma membrane.
\end{abstract}

\section{Introduction}

The boar spermatozoon has been developed as a useful model for the characterization of sperm surface molecules since sperm plasma membranes and plasma membrane proteins (PMPs) can be obtained in large quantities (Gillis, Hook, Peterson, Russell \& Freund, 1978; Peterson, Russell, Bundman \& Freund, 1980; Russell, Peterson \& Russell, 1983b; Kaplan, Russell, Peterson \& Martan, 1984; Hunt et al., 1985; Peterson \& Russell, 1985). The development of methods to capacitate boar spermatozoa in vitro (Nagai, Niwa \& Iritani, 1984; Cheng, Moor \& Polge, 1986) has increased the usefulness of this model since it is now possible to describe the origin, organization and function of referenced PMPs (Russell, Peterson, Russell \& Hunt, 1983a; Saxena, Russell, Saxena \& Peterson, 
1986a; Saxena et al., 1986b). In this report we describe the use of pig oocytes matured in vitro to study fertilization in this species and monoclonal antibody tags to major boar sperm PMPs to describe changes in surface organization that appear to accompany capacitation.

\section{Materials and Methods}

Collection of spermatozoa and sperm plasma membranes. Spermatozoa were collected from the caput and cauda epididymidis of freshly slaughtered boars (Russell, Peterson, Hunt \& Strack, 1984). Cauda spermatozoa are initially immotile but the percentage motility increases to more than $90 \%$ after dilution in a Hepes buffer (Peterson, Russell \& Hunt, 1984). This buffer (designated in the text as Hepes-BSA) contained the following constituents (mM): sodium [ $N$-2-hydroxypiperazine]$N$-ethane sulphonate (Hepes) (20); $\mathrm{NaCl}(95) ; \mathrm{K}_{2} \mathrm{HPO}_{4}$ (1); sucrose (60); $\mathrm{MgCl}_{2}$ (1); $\mathrm{CaCl}_{2}$ (1·5); glucose (5); sodium pyruvate (5); bovine serum albumin (Sigma, St Louis, MO; RIA grade, $5 \mathrm{mg} / \mathrm{ml}$ ). The $\mathrm{pH}$ was $7 \cdot 4$. Motile spermatozoa showed vigorous forward progression. Caput spermatozoa were also immotile when isolated but in the presence of caffeine $(10 \mathrm{~mm})$ developed a circular swimming pattern. These criteria were used to establish the viability of isolated cells used in localization experiments. In these experiments spermatozoa were used directly (without caffeine). Ejaculated spermatozoa (sperm-rich semen fraction) were obtained as described earlier (Gillis et al., 1978). Seminal plasma was obtained from semen samples collected in the presence of the antiprotease phenylmethylsulphonyl fluoride (PMSF, $0 \cdot 2 \mathrm{mM}$ ). Seminal plasma was separated from spermatozoa by centrifuging semen twice at low speed $(1500 \mathrm{~g})$ and then centrifuging the supernatant in an air fuge $(100000 \mathrm{~g}, 10 \mathrm{~min})$. Plasma membrane vesicles were prepared by nitrogen cavitation as described previously (Peterson et al., 1980). These earlier studies and those of Kaplan et al. (1984) have established the purity of these vesicles.

Preparation of monoclonal antibodies. The antibodies used for this study were prepared from PM vesicles and isolated PM proteins as described by Saxena et al. (1986a). Antibodies, expressed in ascites fluid, were used in localization studies. ELISA assays, carried out as described by Saxena et al. (1986b), showed that all monoclonal antibodies used in this study had end points (mid-points of titration curves) at least 500-fold greater in dilution than controls (non-sperm specific ascites). Each monoclonal antibody reacted to a unique species in one- and two-dimensional Western blots of PM proteins transferred to nitrocellulose.

Fluorescence labelling assays. Indirect fluorescence labelling of fixed and live cells was carried out also as described by Saxena et al. (1986a). Ascites fluid containing the monoclonal antibody was used as primary antibody. Biotinylated goat anti-mouse IgG was used as secondary antibody and third-layer labelling used avidin-conjugated fluorescein isothiocyanate. As described by Saxena et al. (1986a), experiments were carried out using different methods of specimen preparation to minimize labelling artefacts. Cells were directly fixed with $3 \%$ paraformaldehyde before labelling. Patterns obtained in this manner were compared with cells incubated live with primary antibody and then fixed with cells after addition of secondary antibody. In turn each treatment was carried out at 4 and at $37^{\circ} \mathrm{C}$ (see Saxena et al., 1986a). Other controls (omission of primary antibody, omission of secondary antibody) are also described by Saxena et al. (1986a).

Capacitation and fertilization in vitro. Ejaculated boar spermatozoa were capacitated according to the method described by Cheng et al. (1986). Semen was diluted 1:1 (v/v) in Hepes-BSA and centrifuged; spermatozoa were washed twice $(1000 \mathrm{~g})$ in this buffer before being suspended in tissue culture medium 199 (TCM 199; Gibco, NY) containing Earl's salts and glutamine. Sodium bicarbonate $(2 \cdot 2 \mathrm{~g} / \mathrm{l})$ was added and the $\mathrm{pH}$ was $7 \cdot 4$. After two washes in this medium (centrifugation at $1000 \mathrm{~g}$ and resuspension in the same medium), the pellet was resuspended in supplemented TCM 199 to a sperm concentration of $2 \times 10^{8}$ cells per ml. The complete medium contained the following constituents: $88 \mathrm{ml} \mathrm{TCM} \mathrm{199;} 12 \mathrm{ml}$ fetal calf serum (Gibco); $2.3 \mathrm{~g}$ bovine serum albumin (Sigma, 
RIA grade); 2.9 mm-calcium lactate; $10 \mathrm{~mm}$-Hepes; $7.5 \mathrm{mg}$ penicillin G; and $5 \mathrm{mg}$ streptomycin. The $\mathrm{pH}$ was $7 \cdot 8$. For some experiments the calcium lactate was replaced by $2.9 \mathrm{mm-EGTA}$. Salt solutions were sterilized by filtration through $0.45 \mu \mathrm{m}$ Millipore filters before use. The sperm suspension was covered with paraffin oil and incubated at $37^{\circ} \mathrm{C}$ for different periods (depending on the design of particular experiments). Spermatozoa were then diluted in supplemented TCM 199 $(\mathrm{pH} 7 \cdot 4)$ to $10^{6}$ cells $/ \mathrm{ml}$ and placed in tissue culture plates $(0.5 \mathrm{ml})$. About 20 oocytes matured in vitro (see below) were added per plate. The percentage sperm motility was evaluated subjectively by light microscopy. A subjective estimate of the percentage of spermatozoa showing activated or a whiplash pattern of motility was made at the same time. Eggs were incubated for an additional $48 \mathrm{~h}$ before being removed, stained and examined for the presence of pronuclei, enlarged sperm heads or embryos.

Staining of oocytes. A trypan-Hoechst nuclear staining procedure developed by Pursel \& Wall (1984; V. G. Pursel, personal communication) for staining embryos was used in all experiments. The procedure is as follows. Slides are cleaned with ethanol and then siliconized (Sigmacote; Sigma); trypan blue ( $10 \mu \mathrm{l}$ of $0 \cdot 1 \%$ solution in $2.3 \%$ sodium citrate) is placed on the slide and eggs are pipetted into the drop. After $3 \mathrm{~min}$ at room temperature, the stain is aspirated. A $10 \mu$ drop of Hoechst stain (Hoechst 3342, Sigma) is placed on the slide warmed to $37^{\circ} \mathrm{C}$ on a slide warmer. The stain is prepared by adding $1 \mathrm{mg}$ Hoechst stain per $\mathrm{ml}$ double-distilled water. Just before use, $10 \mu \mathrm{l}$ of this solution are added to a solution containing $0.25 \mathrm{ml}$ ethanol, $0.75 \mathrm{ml}$ and $2.3 \%$ sodium citrate. After $3 \mathrm{~min}$, the stain is removed and a drop of Aqua-Mount (Lerner Laboratories, New Haven, CT) is added and a cover slip is placed over the stained eggs. Slides were examined in the dark with a Nikon Optiphot fluorescence microscope using a $340 \mathrm{~nm}$ excitor filter and a $430 \mathrm{~nm}$ barrier filter. Photographs were obtained using Tri-X-Pan film ASA 400 (exposure time $1 \mathrm{~min}$ ) and developed in Diafine (Kodak; Rochester, NY).

Maturation of oocytes. Oocytes were obtained from fresh ovaries (no later than $3 \mathrm{~h}$ after slaughter). Ovaries were maintained in warm $\left(37^{\circ} \mathrm{C}\right)$ saline during transit to the laboratory. Oocytes from medium to large follicles ( $\sim 5 \mathrm{~mm}$ diameter) were aspirated into sterile TCM 199 at $37^{\circ} \mathrm{C}$ using a 21 -gauge sterile needle and syringe. Gentle suction was provided by a vacuum pump. Oocytes were washed by transferring them twice through TCM $199(0.5 \mathrm{ml})$ in sterile tissue culture dishes (Falcon; Oxnard, CA). The eggs (20-25 per dish) were then transferred to supplemented TCM 199 and covered with paraffin oil. Manipulations were carried out with sterile drawn-out glass pipettes using a dissecting microscope. Dishes were placed in an incubator at $37^{\circ} \mathrm{C}\left(5 \% \mathrm{CO}_{2}\right.$, $95 \%$ humidified air) for $48 \mathrm{~h}$ to complete maturation. The number of live eggs, after maturation, was determined by trypan blue exclusion. Oocytes that displayed any morphological abnormalities (retracted cytoplasm, fragmented zonae) were not selected.

Preparation of sperm extract 'stop solution'. To prevent continued penetration of eggs after specified intervals by excess spermatozoa in some experiments, a method was developed to prevent spermatozoa from binding to eggs. This method was based on an earlier observation that supernatants from spermatozoa, centrifuged at high speed, inhibited spermatozoa from attaching to eggs (Peterson et al., 1981). Ejaculated spermatozoa $\left(5 \times 10^{8}\right.$ cells) were washed three times in PBS and resuspended in Hepes-BSA. Spermatozoa were sonicated at ice-bath temperature for $2 \mathrm{~min}$ (15 sec intervals at maximum power; Branson Sonifier) and then centrifuged at $12000 \mathrm{~g}$ (Eppendorf). The supernatant was removed and sterilized by Millipore filtration. Oocytes that had been incubated with spermatozoa were washed once in PBS and added to $50 \mu$ of the 'stop solution'. After 20 min, the oocytes were washed twice in PBS before being placed again in supplemented TCM 199 to complete the $48 \mathrm{~h}$ fertilization interval. Spermatozoa bound to eggs were removed by this treatment and fresh spermatozoa failed to bind to the eggs which still were viable as described in the text. 
Preparation of monoclonal antibodies. Monoclonal antibodies were prepared as previously described (Saxena et al., 1986a; Hunt et al., 1985). Western blot assays from two-dimensional PAGE gels were used to determine the specificity of these antibodies.

Three of the monoclonal antibodies used were directed against PM proteins that have been referenced in two-dimensional surface maps of the boar spermatozoon (Russell et al., 1983a).

\section{Results}

Figure 1 shows the relation between the time spermatozoa were incubated in supplemented TCM 199 (capacitation medium) and the percentage of eggs that were penetrated. For these experiments, a sperm extract ('stop solution'; see 'Methods') was used to stop further penetration of eggs and dislodge spermatozoa bound to the outer layer of the zona. The 'stop solution' was added $2 \mathrm{~h}$ after spermatozoa (previously incubated in capacitation medium) were diluted in the medium containing matured eggs. The percentage of live eggs ( $\sim 60 \%$ of the total number of eggs matured) and the number of live eggs fertilized were not affected by this treatment. Figure 2(a) is a photograph of a fertilized egg, stained with Hoechst-trypan and exposed to 'stop solution' after incubation with capacitated spermatozoa for $2 \mathrm{~h}$. Eggs were washed and returned to complete medium for an additional $46 \mathrm{~h}$. Figure 2(b) shows a fertilized egg in which the 'stop solution' had not been used. The removal of large numbers of spermatozoa, ordinarily bound to such eggs, by the 'stop solution' is obvious from this photograph.

In Fig. 1 the point at 0 time indicates that about $20 \%$ of eggs were fertilized without incubating spermatozoa at high concentration in capacitating medium. Simply diluting spermatozoa to $\sim 10^{6}$ cells $/ \mathrm{ml}$ in capacitating medium was sufficient to fertilize this small percentage of eggs. However, the use of diluted spermatozoa incubated for longer periods in capacitating medium did not increase significantly the percentage of eggs fertilized. Maximum fertilization $(\sim 60 \%)$ was achieved after $4 \mathrm{~h}$ of incubating spermatozoa at higher concentration $\left(2 \times 10^{8} / \mathrm{ml}\right)$. Whiplash (activated) motility was observed as soon as $1 \mathrm{~h}$ after incubation in capacitation medium and, after $4 \mathrm{~h}$ in this

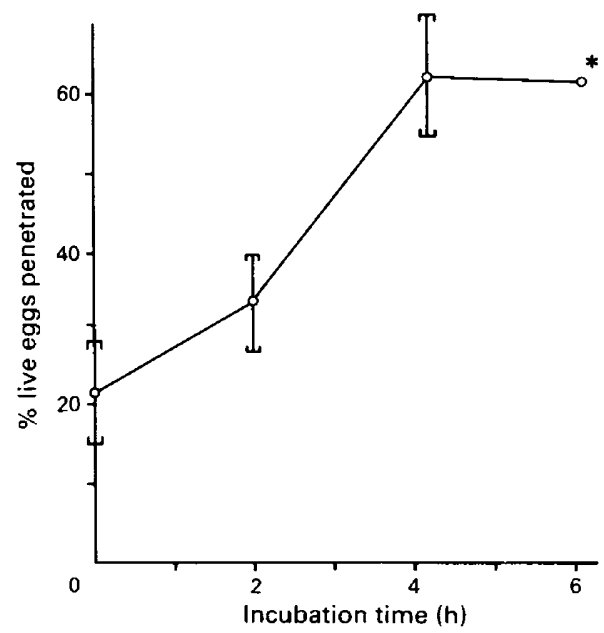

Fig. 1. Effect of capacitation period on the percentage fertilization of pig eggs matured in vitro. Percentages are given for eggs that were viable after incubation with spermatozoa for $48 \mathrm{~h}$. Some eggs were fertilized by spermatozoa that at high sperm concentrations had not been incubated in capacitation medium (see text). Data points are averages ( \pm s.e.m.) of assays carried out in triplicate. The assays at $6 \mathrm{~h}$ (asterisk) were carried out in duplicate. 

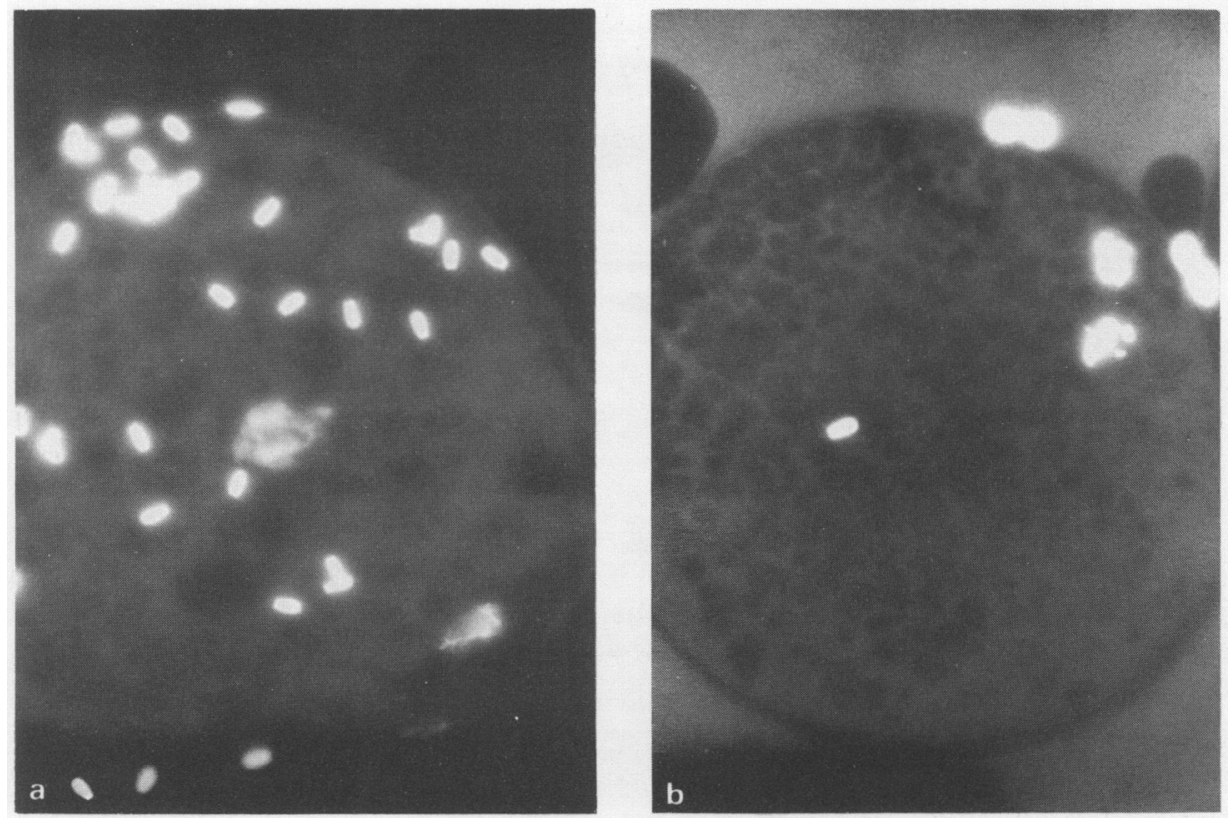

Fig. 2. Effect of 'stop' extract on the number of spermatozoa bound to pig eggs 'matured in vitro'. (a) No 'stop' solution added to eggs. (b) 'Stop' solution added to eggs $2 \mathrm{~h}$ after insemination. Eggs were washed and incubated for another $46 \mathrm{~h}$.

medium, occurred in about $40 \%$ of all cells. When spermatozoa were incubated in Hepes-BSA the percentage of eggs penetrated after $4 \mathrm{~h}$ was significantly lower $(\sim 20 \%)$ and activated motility was observed in only a small percentage $(\sim 10 \%)$ of cells. No fertilization or activated motility was observed when spermatozoa were incubated in PBS.

\section{Effect of capacitation buffer on localization of surface antigens}

Four monoclonal antibodies were used to detect changes in surface organization during incubation of spermatozoa in capacitation buffer. These antibodies are designated 1F3, 3D 3, 4D 10 and 5D7. 4D10, 1F3 and 5D7 are specific for PM proteins $2 \cdot 0,4 \cdot 85$ and 5.0, respectively; these are shown in the two-dimensional PAGE tracing of cauda sperm PM proteins (Fig. 3). The PM proteins are referenced according to Russell et al. (1983a). Antibody 3D3 has been shown to be specific for a PM protein with a molecular weight near 80000 but has not been referenced in two-dimensional PAGE surface maps. Earlier studies (Russell, Montag, Hunt \& Peterson, 1985) have shown that PM protein 5.0 is solubilized from isolated PM vesicles only by detergents and appears to be integral to the membrane. PM proteins 2.0 and 4.85 are extracted by hypotonic and hypertonic salts but are also readily extracted by detergents. These PM proteins do not appear to be inserted into the PM bilayer but may be associated with the bilayer by hydrophobic interactions. The relationship of the PM protein of $M_{\mathrm{r}} 80000$ to the bilayer has not been established. Figure 4 shows pattern changes for each monoclonal antibody tested on ejaculated spermatozoa at 0 and $4 \mathrm{~h}$ after capacitation in TCM 199 in the presence and absence of calcium. The change in localization of antibody 5D7 (against the major PM protein 5.0) during capacitation of ejaculated spermatozoa is striking in that the fluorescence of the PM overlying most of the sperm head disappeared in the majority of cells, and fluorescence appeared almost entirely in the flagellum. This change occurred 


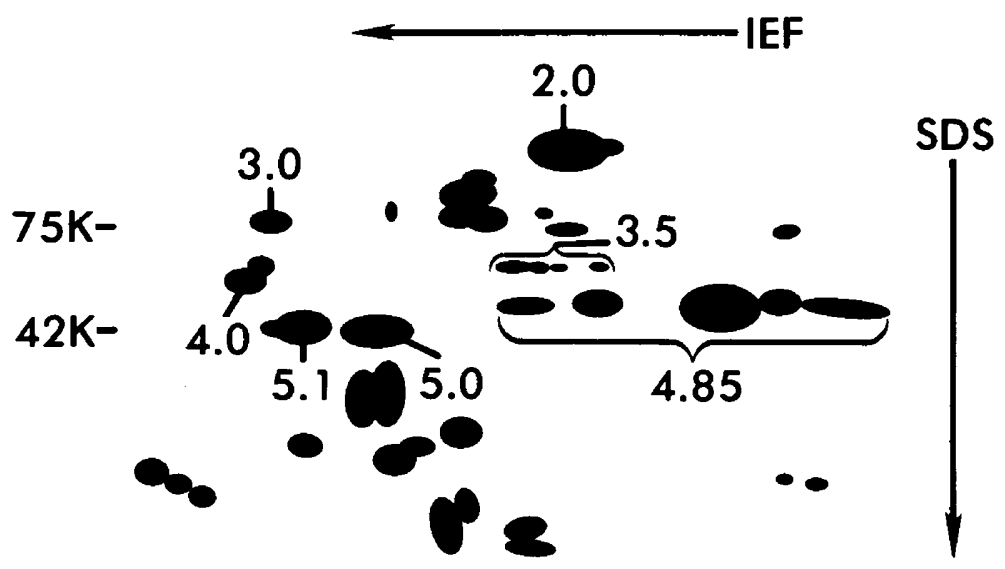

Fig. 3. Two dimensional PAGE reference pattern for boar sperm plasma membrane (PM) proteins. The tracing shows only major (Coomassie-stained) PM proteins. A complete reference map is given by Russell et al. (1983a).

gradually during the course of $4 \mathrm{~h}$ and was more rapid in the presence of calcium. Patchy fluorescence developed in most cells during the first $2 \mathrm{~h}$ (not shown) and after this time about $10 \%$ of all cells had lost fluorescence in the head PM. At the end of $4 \mathrm{~h}$ more than $70 \%$ of all cells (Fig. $4 \mathrm{~b}$ ) had lost fluorescence over most of the head PM. This loss of fluorescence was inhibited when calcium was chelated with EGTA (Fig. 4c).

Localization changes occurring with antibody 3D3 (against the protein of $M_{\mathrm{r}} 80000$ ) were similar to those for antibody 5D7 and after $4 \mathrm{~h}$ most spermatozoa had lost fluorescence over most of the sperm head PM, and fluorescence appeared only on the flagellar PM, but a few spermatozoa showed whole cell fluorescence (Fig. 4d). This change was almost completely inhibited by the presence of EGTA (Fig. 4f). In all cases the changes described were not affected by the manner in which cells were prepared for fluorescence assay. Similar patterns were observed when cells were prefixed before labelling or when cells were labelled first with primary antibody and then fixed. Patterns were also unaffected by varying the concentration of primary antibody (1:20-1:500). These localization changes did not occur in PBS which failed to capacitate spermatozoa.

Antibodies 4D10 and 1 F3 (against PM proteins 2-0 and 4.85) showed a less striking variation during incubation in capacitation buffer. A patchy, dull fluorescence, initially observed with antibody $4 \mathrm{D} 10$ on the PM overlying the head, gradually changed during the capacitation period to a pattern in which, for many cells, fluorescence in the principal segment was observed only on the edges of the head PM (Fig. 4h). This change occurred to a much smaller extent when EGTA was used to chelate calcium (Fig. 4i). The fluorescence pattern with antibody $1 \mathrm{~F} 3$ initially showed localization over the anterior region of the head PM; this pattern gradually changed during the capacitation period to a patchy fluorescence and the appearance of fluorescence in the flagellum (Fig. 4k, 1). Patchy fluorescence in the head PM was not observed in the absence of calcium (Fig. $4 \mathrm{~m}$ ).

Fig. 4. Effect of incubating spermatozoa in capacitation medium on the fluorescence patterns developed with monoclonal antibodies (5D7, 3D3, 4D10, 1F3) to different PM proteins. For many cells tagged with antibodies 5D7 and 3D3 (Figs 4b, e) fluorescence over the entire head PM is lost after $4 \mathrm{~h}$ incubation in capacitation medium (arrows). Fluorescence appears in the flagellum for 5D7 and is retained in the flagellum for 3D3. These changes occur much less rapidly in the absence of calcium. Loss of fluorescence in the head PM for 4D10 and 1F3 does not occur; rather, fluorescence disperses to the rim of the principal segment of the head PM (4D10) or disperses into patches (1F3). An enlarged photograph of the patched pattern for $1 F 3$ is shown in Fig. 4(k). 


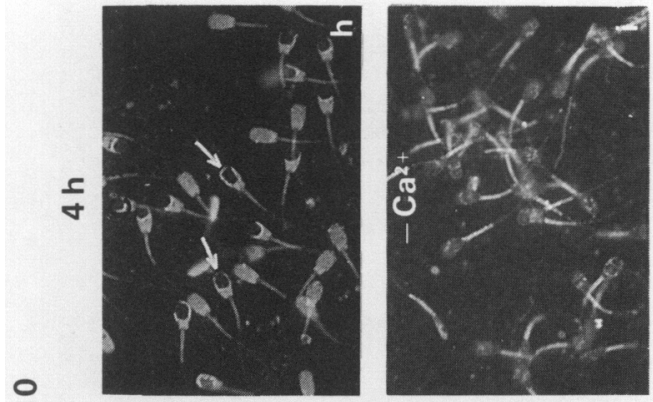

$\frac{0}{2}$
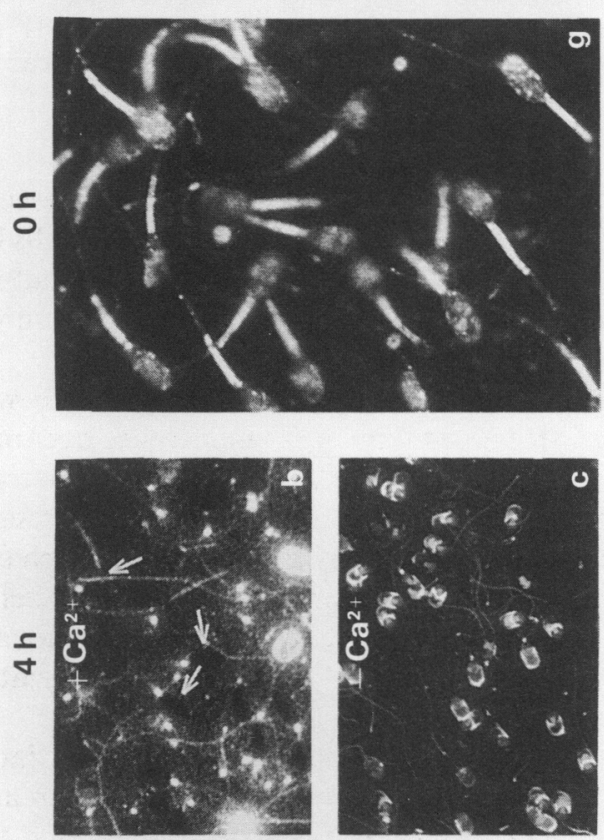

กิ

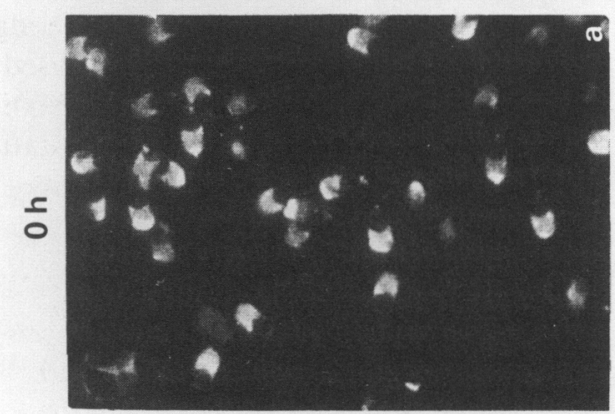

$\stackrel{m}{\longleftarrow}$
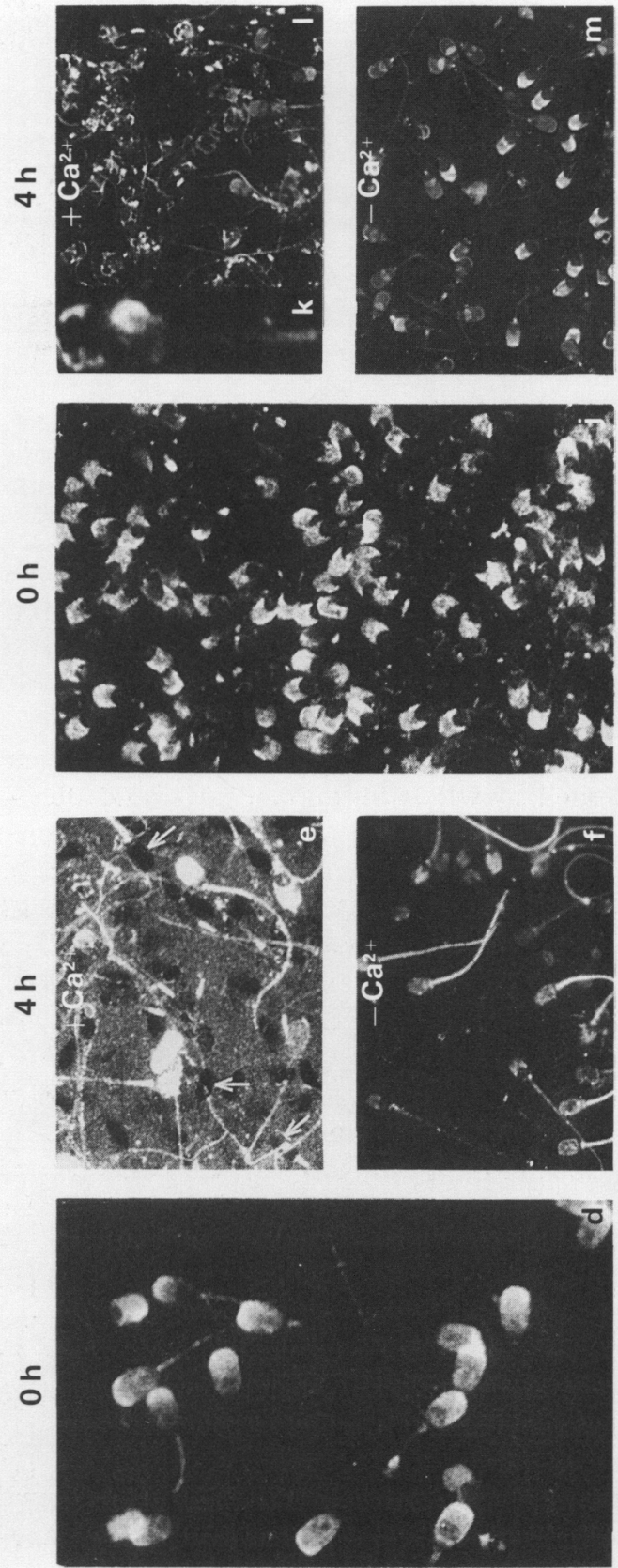

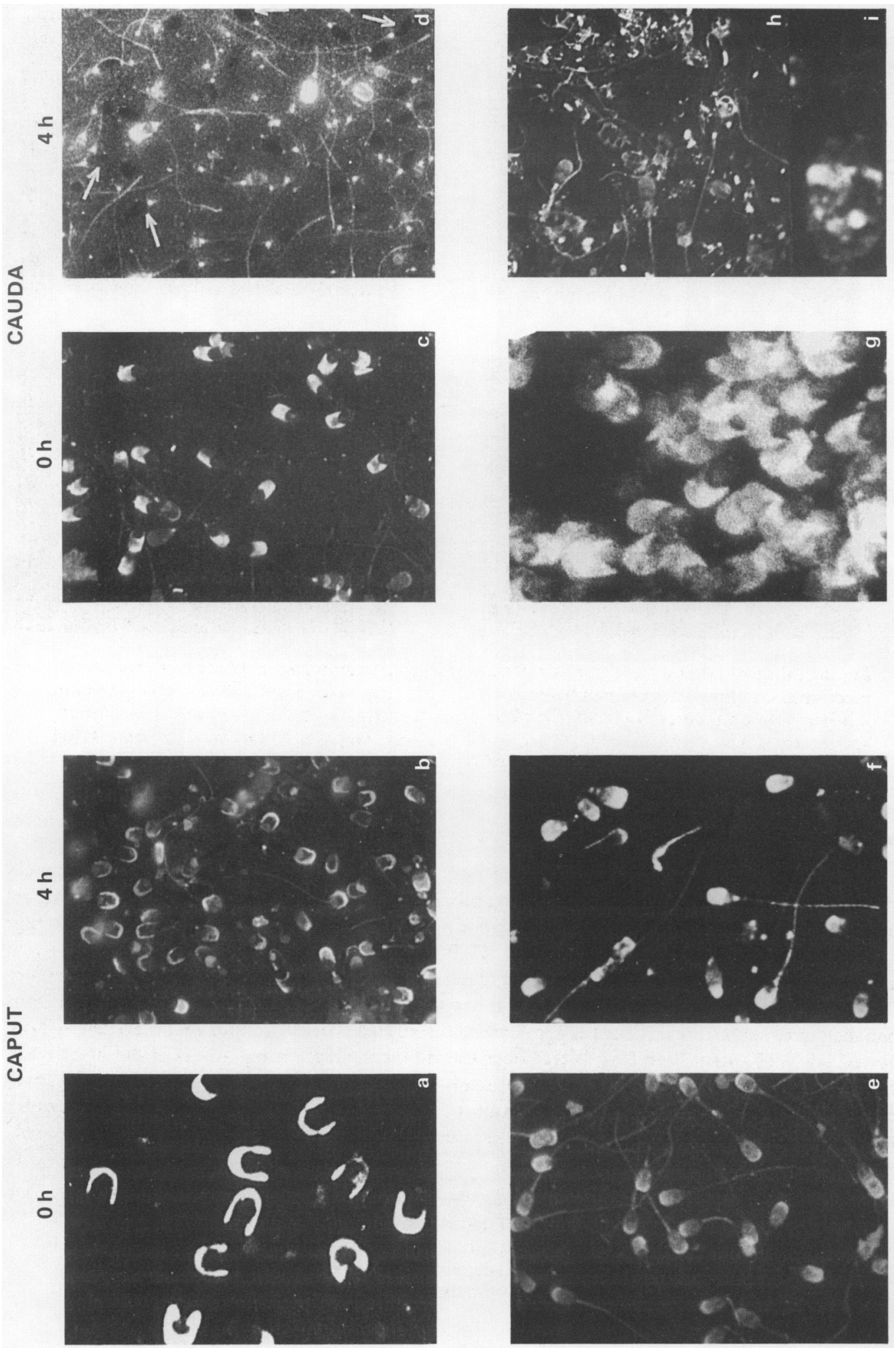

ดे
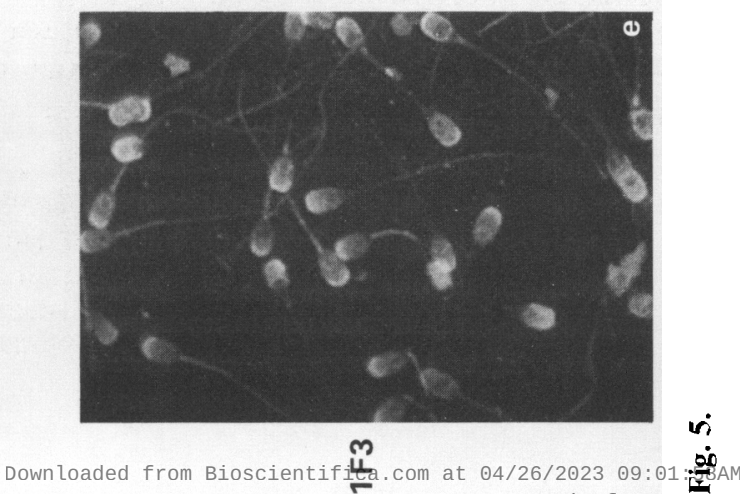

via free access 

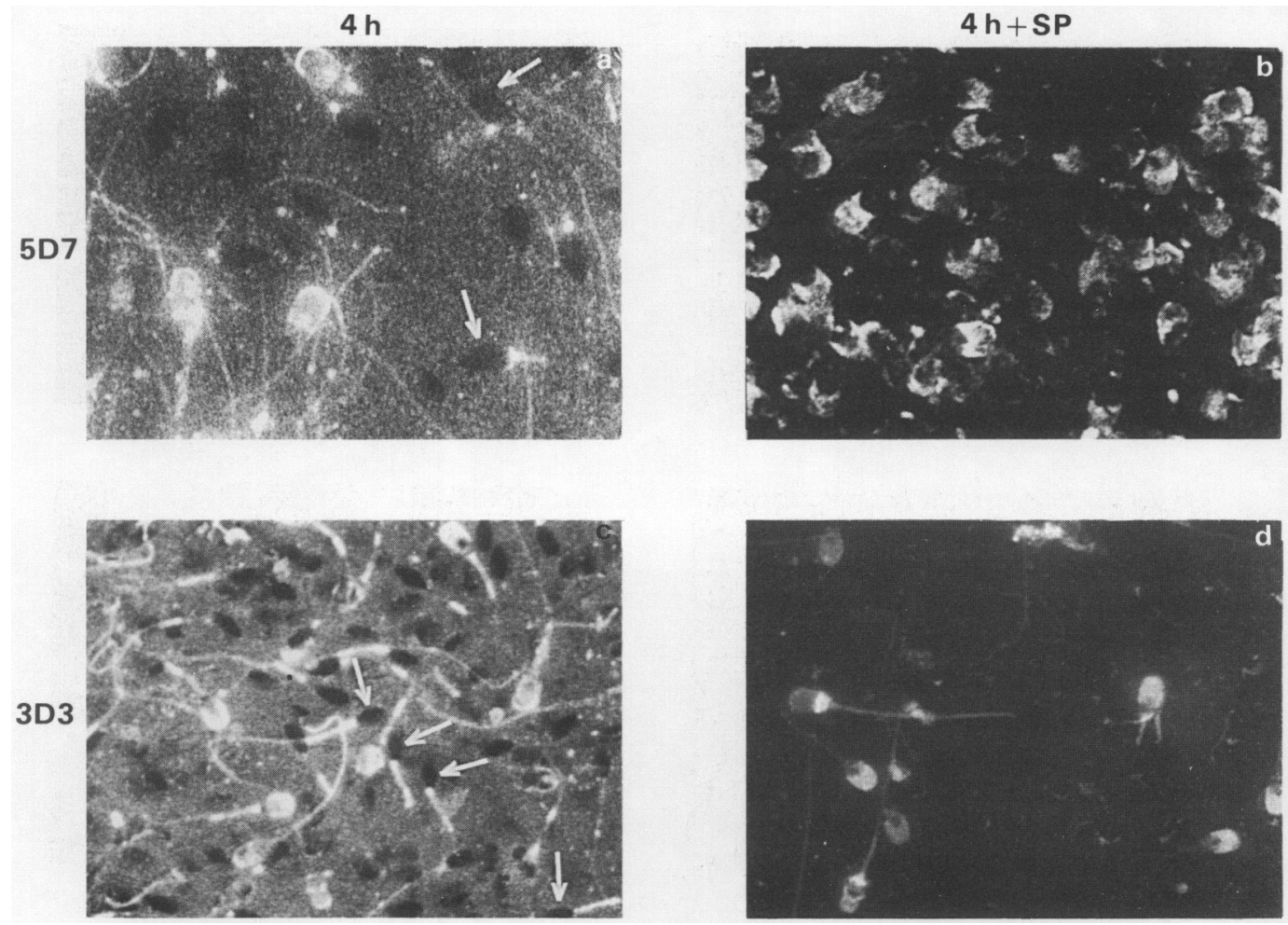

Fig. 6. Effect of adding fresh seminal plasma (SP) to spermatozoa incubated in capacitating medium. (a, c) Spermatozoa incubated for $4 \mathrm{~h}$ in supplemented TCM 199 and then fixed and labelled with antibodies 5D7 and 3D3. The absence of fluorescence in the head PM of many spermatozoa is easily discerned (a, c, arrows). Fresh seminal plasma was added to 'capacitated' spermatozoa, and incubation was continued for another $2 \mathrm{~h}$ before fixation and labelling.

\section{Localization changes in epididymal spermatozoa}

When epididymal spermatozoa were incubated in capacitating medium quantitative differences in fluorescence pattern changes between caput and cauda spermatozoa were observed. This was most apparent for antibody 5D7 as shown in Fig. 5(a-d). Cauda spermatozoa which readily fertilize eggs in vitro when capacitated in TCM 199 (E. J. C. Polge, personal communication) showed pattern changes similar to those for ejaculated spermatozoa after capacitation for $4 \mathrm{~h}$ (Fig. $5 \mathrm{~d}$ ). The presence of calcium was not as critical to this change as in ejaculated spermatozoa and fluorescence patterns changed rapidly in the presence of EGTA. Strikingly, only a minority of caput spermatozoa $(\sim 20 \%)$ showed a loss of sperm head PM fluorescence during the incubation regardless of the presence or absence of calcium (Fig. 5b). Caput spermatozoa have been shown to fertilize superovulated eggs at very low efficiency compared to cauda spermatozoa (Holtz \& Smidt, 1976). Pattern changes for antibody $1 \mathrm{~F} 3$ were similar to those occurring in ejaculated spermatozoa with patchy fluorescence

Fig. 5. Change in fluorescence patterns for epididymal spermatozoa tagged with antibody 5D7 and $1 F 3$ incubated in capacitating medium. Head PM fluorescence is lost after capacitation in many cells tagged with antibody 5D7 (Fig. 5d, arrows) and for both antibodies fewer caput spermatozoa show characteristic pattern changes compared to cauda spermatozoa. The patchy fluorescence which develops on cauda PM incubated with antibody IF3 is also shown in the enlarged photograph (Fig. 5i). 

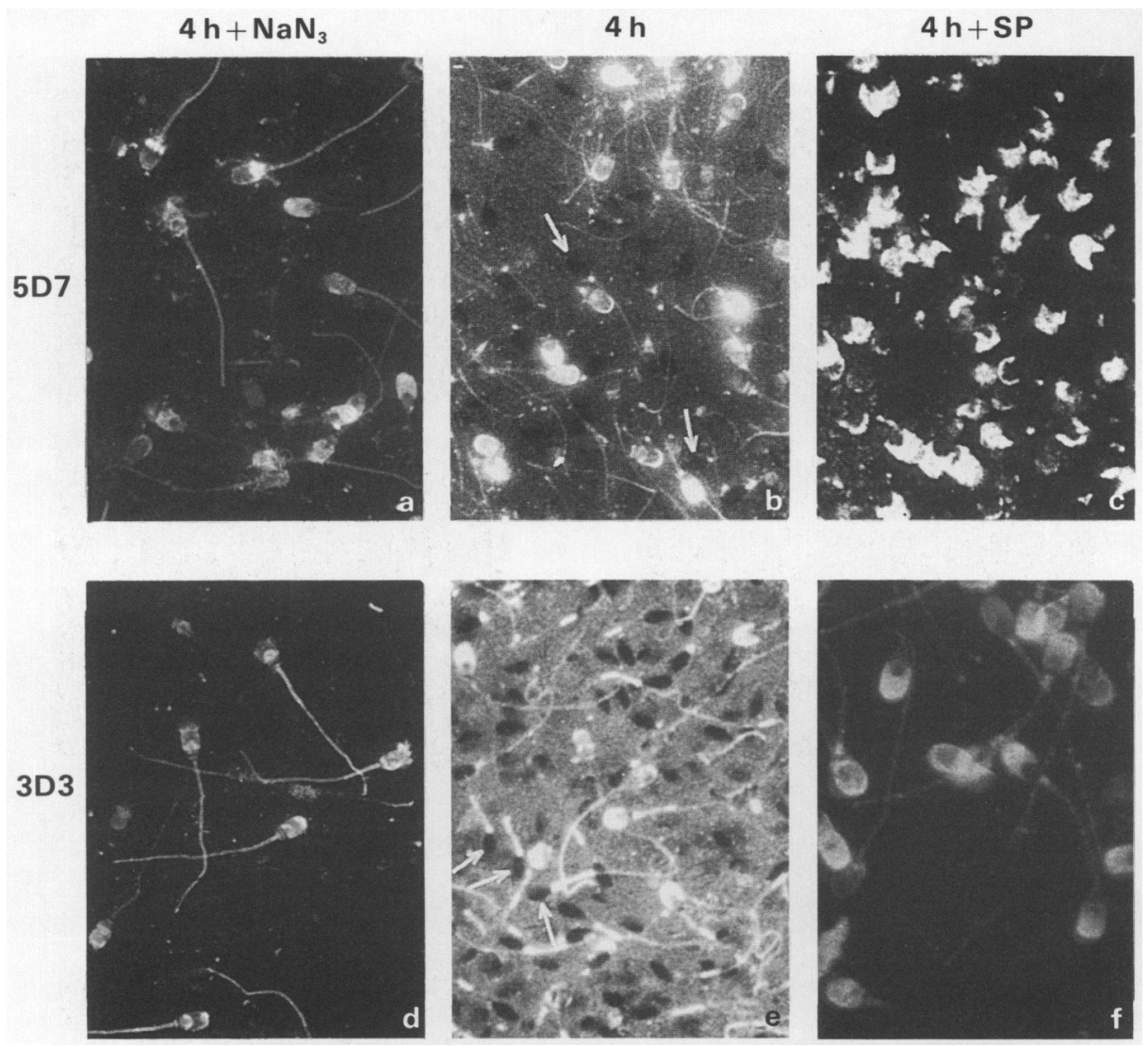

Fig. 7. Effect of seminal plasma and sodium azide on spermatozoa tagged with antibodies 5D7 and 3D3 and incubated for $4 \mathrm{~h}$ in capacitation medium. (b, e) Fluorescence pattern for tagged live spermatozoa after $4 \mathrm{~h}$ incubation in capacitation medium. In most cells antigen-antibody complexes had migrated into the flagellar PM. (a, d) Effect of the addition of $0.01 \mathrm{M}-\mathrm{NaN}_{3}$ to tagged, 'capacitated' spermatozoa. Antigen-antibody complexes appear throughout the sperm head after a short incubation $(30 \mathrm{~min})$. (c, $f$ ) Effect of the addition of seminal plasma $(1: 1, v / v)$ to tagged 'capacitated' spermatozoa. Note that fluorescence develops again at the head PM and that the patterns are similar to those observed in spermatozoa before capacitation (see Fig. 4).

seen over most of the head PM. This change also occurred in a smaller percentage of caput spermatozoa (Fig. 5e-i).

\section{Reversal of fluorescence changes by seminal plasma}

When fresh seminal plasma was added to spermatozoa capacitated in TCM 199, patterns observed before capacitation were restored for each of the four antibodies used (see Fig. 6a, b; 6c, d). Only fresh seminal plasma restored the patterns; when seminal plasma was denatured by boiling, fluorescence patterns did not change. These observations were interpreted to mean that either seminal plasma proteins restored antibody accessibility to antigenic sites that became masked during capacitation, or that antigens present in the head PM changed location during capacitation. Since the loss of fluorescence on the PM overlying the entire sperm head was the most striking change observed with the monoclonal antibodies used, we attempted to determine whether incubation in TCM 199 was masking the antigenic sites in the sperm head PM or whether antigens were migrating out of the head PM. To do this, live spermatozoa were first labelled with antibody 5D7 or antibody 3D3, 
CD
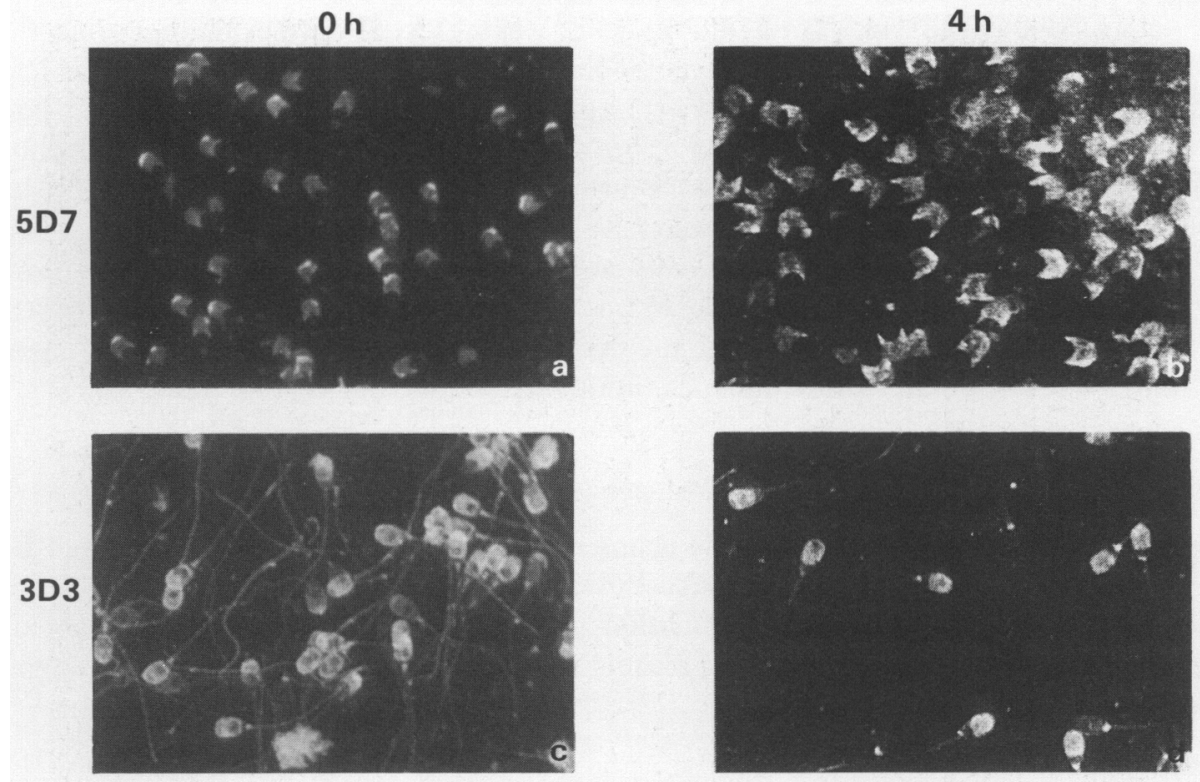

$C D+S P$

$4 \mathrm{~h}$
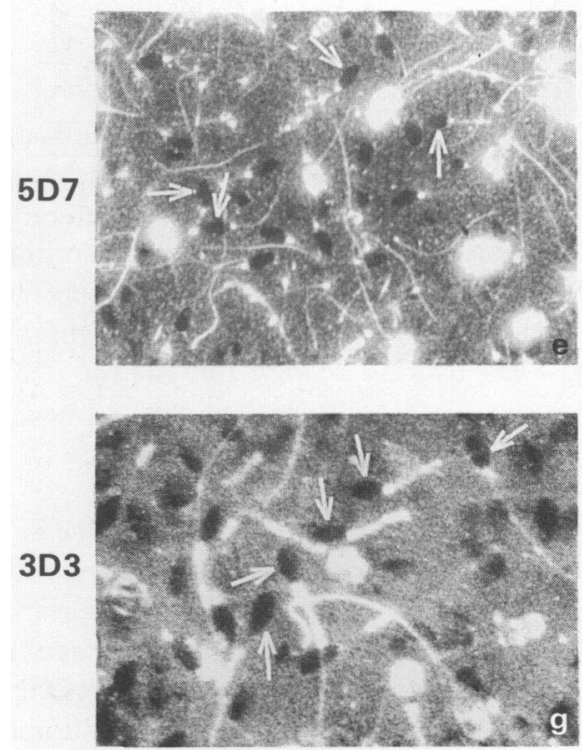

4 h cap. +2 h SP \& CD
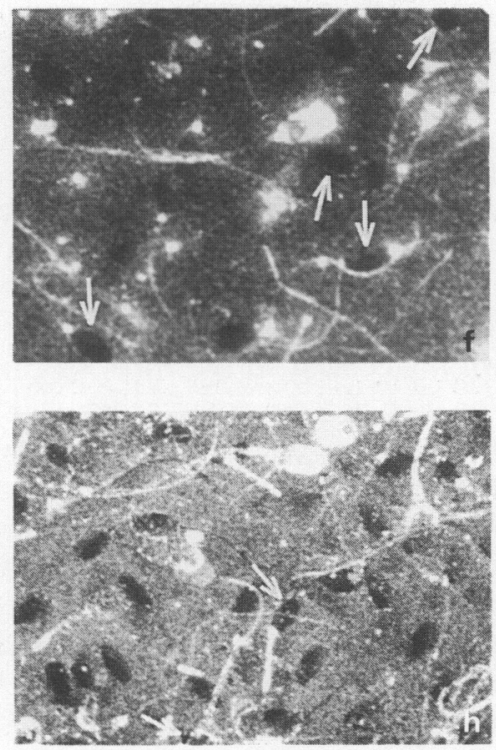

Fig. 8. Effect of $50 \mu \mathrm{M}$ cytochalasin $\mathrm{D}$ (CD) on the fluorescence patterns of antibodies 5D7 and 3D 3 before and after incubation in capacitation medium. (a-d) Spermatozoa were tagged with antibodies before incubation. Fluorescence is retained in the head PM with both antibodies. (e-h) The inclusion of seminal plasma, which induced migration of antibody-antigen complexes from the flagellar PM after incubation in capacitation medium failed to induce movement of these complexes back into the head PM in the presence of $\mathrm{CD}$. Arrows point to some of the numerous cells that still show an absence of fluorescence in the head PM (compare to Figs $7 \mathrm{c}$ and $7 \mathrm{f}$ ). 
washed and then incubated in TCM 199 for $4 \mathrm{~h}$. When these cells were subsequently fixed and labelled with secondary antibody, most fluorescence was observed only in the flagellum (Fig. 7), indicating that the antibodies (and the antigen bound to them) had migrated out of the sperm head (Fig. $7 \mathrm{~b}$, e) where they were first located. Furthermore, when fresh seminal plasma was added to these 'capacitated cells', fluorescence again appeared in the head PM (Fig. 7c, f) indicating that antigen-antibody complexes had migrated back into the head region. Antigen migration into and out of the head PM appeared to depend, at least in part, on energy metabolism since brief incubation in $\mathrm{NaN}_{3}$ (30 min) of spermatozoa tagged with antibody 5D7 or 3D3 and capacitated also resulted in the migration of antigen--antibody complexes back into head PM (Fig. 7a, d). PM protein 5.0 (antibody 5D7) is a major integral membrane protein and removal of integral head membrane proteins may be a prominent feature of capacitation (see below).

\section{Effect of cytochalasin D on PM protein 5.0 movement during capacitation}

Cytochalasin D completely blocked the change of fluorescence localization observed for PM protein 5.0 and that of $M_{\mathrm{r}} 80000$ during capacitation (Fig. 8a, b; 8c, d). More strikingly, antigens that had migrated into the tail after incubation in capacitation medium were prevented from migrating back into the head when seminal plasma was added in the presence of this antagonist of actin polymerization (Fig. 8e-h).

\section{Discussion}

The use of eggs matured in vitro, as described in this report, has the advantage that many eggs can be used in a single experiment. About $50 \%$ of the eggs selected were viable up to $48 \mathrm{~h}$ after maturation and, of these, nearly $60 \%$ were penetrated by capacitated spermatozoa. The use of Hoechst-trypan stain in conjunction with a sperm extract to remove an excess number of bound spermatozoa shortened the time required to fix and stain eggs for microscopic examination. Eggs can be examined within a few hours after the end of an experiment compared to the several days required with older alcohol-acetic acid-acetorcein staining procedures (Polge \& Dziuk, 1965). The nature of the components in the sperm extract used to prevent additional binding of spermatozoa to the zona or to remove excess spermatozoa from the surface of the zona has not been determined but the extract probably consists primarily of hydrolytic enzymes from the acrosome. Binding sites for sperm attachment to the zona appear to be removed by this extract, but for the period of incubation used in these experiments, eggs remained viable. Previously, we had shown by scanning electron microscopy that such sperm extracts modify the structure of the zona pellucida (Russell, Peterson \& Freund, 1979).

Under the conditions of capacitation described in the report, the location of 4 surface antigens ( 3 of which are located on major plasma membrane proteins) is markedly changed. A relationship between these pattern changes and capacitation is suggested by the following observations. (1) Patterns changed gradually and were stabilized after a period in which the percentage fertilization and activated motility was maximum. (2) Similar localization changes were not observed in PBS which failed to capacitate spermatozoa. (3) Pattern changes were the same for cauda and ejaculated spermatozoa which fertilize eggs with a high efficiency in vitro (this paper) and in vivo (Holtz \& Smidt, 1976), but only a small percentage of caput spermatozoa, which fertilize eggs with a low efficiency in vivo, and which fail to bind to eggs in vitro (Peterson et al., 1984), showed localization changes similar to that observed with cauda sperm. (4) Fresh seminal plasma, which prevents fertilization by previously capacitated spermatozoa, restores original localization patterns. We wish to make it clear, however, that while we observe that specific fluorescence changes occur under capacitating conditions, we cannot yet state that the pattern generated is indicative of the capaci- 
tated state. The reversal of the pattern of antigen localization by seminal plasma is particularly striking and, since no changes were observed when seminal plasma was denatured by heat, is apparently the result of an effect of seminal plasma proteins on membrane structure. For two antigens (PM protein 5.0 and the PM protein of $M_{\mathrm{r}} 80000$ ) the proteins apparently migrate out of the head into the tail region. This is observed with spermatozoa fixed before labelling or when live spermatozoa are tagged with antibody and incubated in capacitation buffer, and then labelled with secondary antibody. When seminal plasma is added to capacitated spermatozoa containing antibody bound primarily to the flagellum, incubation in capacitation medium restores fluorescence to the head. It is very unlikely that antigen is released from the sperm tail and then rebinds to the sperm head. PM protein 5.0 is integral to the membrane and its release from the plasma membrane would involve disruption of the bilayer. Our view is that both PM protein 5.0 and the PM protein of $M_{\mathrm{r}}$ 80000 are both integral plasma membrane proteins and migrate out of the head region during capacitation. The pattern changes for PM proteins 2.0 and 4.85 (peripheral) suggest that clearing or dispersal of surface peripheral antigens is also occurring throughout the head PM during the capacitation interval. Clearing of areas in the head plasma membrane and outer acrosomal membrane have been observed for the guinea-pig during capacitation using freeze fracture techniques (Friend, Perrelet, Orci \& Yanagimachi, 1977).

Incubation in capacitating buffer exposes antigenic sites on the flagellum, and, at least for the monoclonal antibodies used in this study, no other changes in specific regions of the flagellum were observed. This exposure of sites is probably due to the removal of seminal plasma proteins which cover the entire ejaculated boar spermatozoon (Russell et al., 1983a). The specific effects of seminal plasma on the mobility and location of head PM surface antigens indicates an interaction of seminal plasma constituents on PM protein within this region of the sperm surface. Mobility and/or the conformation of surface proteins may be directly affected by these specific interactions. Since azide disrupts the restriction in antigen localization induced by incubation in capacitating buffer, energy metabolism is apparently involved in the changes observed.

The 'freezing' of fluorescence patterns for PM protein 5.0 and that of $M_{\mathrm{r}} 80000$ before or after capacitation by the microfilament antagonist cytochalasin D is a striking finding and suggests that cytoskeletal components linked to the plasma membrane play a key role in surface changes during capacitation. Actin and other cytoskeletal components have been shown to be present in mammalian spermatozoa (Clarke \& Yanagimachi, 1978; Tamblyn, 1980; Virtanen, Badley, Paasivuo \& Lehto, 1984). Since PM protein 5.0 has been characterized as an integral plasma membrane protein (Russell et al., 1985) and therefore may penetrate through the bilayer, it is not improbable that this major PM protein is linked to cytoskeletal components. Such transmembrane proteins may transmit environmental information (e.g. binding of seminal plasma proteins to the PM) to cytosol components involved in control of surface organization. The identification of these PM proteins could lead to experimental testing of this hypothesis.

Localization changes associated with capacitation appear to be accelerated by the presence of calcium in ejaculated spermatozoa but not in those from the cauda epididymidis. The reason for this is not clear but calcium-activated enzymic reactions may accelerate changes in surface organization that remove restraints, imposed by seminal plasma components, on the movement of head PM antigens. Boar cauda epididymal spermatozoa do not require any preincubation in capacitation buffer to fertilize superovulated eggs at high efficiency (E. J. C. Polge, personal communication); calcium, therefore, may play a role in removing or countering the decapacitating effects of accessory gland proteins other than those contributed by the epididymis.

Whether the surface changes noted in this study with several major plasma membrane proteins of boar spermatozoa occur with other major PM proteins is under study, but our major effort is to determine how the loss of seminal plasma constituents induce a major reorganization of PM proteins in the sperm head PM. The head PM of the boar spermatozoon appears to become an unfavourable site for the localization of several major antigens. Seminal plasma may affect this localization by direct association with particular surface proteins, by affecting PM protein-cytoskeletal reactions 
and by altering the chemical composition of the lipid bilayer. Determining what seminal plasma proteins produce these effects and how they produce them are questions now under study.

This work was supported by the Lalor and Noyes Foundations and a grant HD 13094 from the National Institutes of Health. We thank Ms Janet Landrie for excellent photographic work, and Dr N. K. Saxena and Dr L. D. Russell for help with antibody preparation.

\section{References}

Cheng, W., Moor, R.M. \& Polge, C. (1986) In vitro fertilization of pig and sheep oocytes matured in vitro. Theriogenology 25, 146, Abstr.

Clarke, G.N. \& Yanagimachi, R. (1978) Actin in mammalian sperm heads. J. exp. Zool. 205, 125-132.

Friend, D.S., Perrelet, A., Orci, L. \& Yanagimachi, R. (1977) Membrane particle changes attending the acrosome reaction in guinea pig spermatozoa. $J$. Cell Biol. 74, 561-577.

Gillis, G., Hook, L., Peterson, R.N., Russell, L.D. \& Freund, M. (1978) Isolation and characterization of plasma membranes from human and boar sperm: a method using nitrogen cavitation. Prep. Biochem. 8, 363-378.

Holtz, W. \& Smidt, D. (1976) The fertilizing capacity of epididymal spermatozoa in the pig. J. Reprod. Fert. 46, 227-229.

Hunt, W.P., Peterson, R.N., Saxena, N.K., Saxena, N., Arthur, R., Russell, L.D. (1985) Isolation of major proteins of boar sperm plasma membranes by preparative gel electrophoresis and localization of a major polypeptide using specific monoclonal antibodies. Prep. Biochem. 15, 9-33.

Kaplan, M., Russell, L.D., Peterson, R.N. \& Martan, J. (1984) Boar sperm cytoplasmic droplets: Their ultrastructure, their numbers in the epididymis and at ejaculation and their removal during isolation of sperm plasma membranes. Tissue and Cell 16, 455-468.

Nagai, T., Niwa, K. \& Iritani, A. (1984) Effect of sperm concentration during preincubation in a defined medium on fertilization in vitro of pig follicular oocytes. J. Reprod. Fert. 70, 271-275.

Peterson, R.N. \& Russell, L.D. (1985) The mammalian spermatozoon: a model for the study of regional specificity in plasma membrane organization and function. Tissue and Cell 17, 769-779.

Peterson, R.N., Russell, L.D., Bundman, D. \& Freund, M. (1980) An evaluation of the purity of boar sperm plasma membranes prepared by nitrogen cavitation. Biol. Reprod. 23, 637-645.

Peterson, R.N., Russell, L.D., Bundman, D. \& Freund, M. (1981) The interaction of living boar sperm and sperm plasma membrane vesicles with the porcine zona pellucida. Devl Biol. 84, 144-156.

Peterson, R.N., Russell, L.D. \& Hunt, W.P. (1984) Evidence for specific binding of uncapacitated boar sperm to porcine zonae pellucidae in vitro. $J$. exp. Zool. 231, 137-147.
Polge, C. \& Dziuk, P. (1965) Recovery of immature eggs penetrated by spermatozoa following induced ovulation in the pig. J. Reprod. Fert. 9, 317-358.

Pursel, V.G. \& Wall, R.J. (1984) Effect of staining with TR ITC on cleavage of pig embryos. J. Anim. Sci. 59 (Suppl. 1), 359-360, Abstr.

Russell, L.D., Peterson, R.N. \& Freund, M. (1979) Morphological observations on the binding of boar sperm to the porcine zona pellucida. Scanning Electron Microscopy J. III, 407-412.

Russell, L.D., Peterson, R.N., Russell, T.A. \& Hunt, W.P. (1983a) Electrophoretic map of boar sperm plasma membrane polypeptides and localization and fractionation of specific polypeptide subclasses. Biol. Reprod. 28, 393-413.

Russell, L.D., Peterson, R.N. \& Russell, T.A. (1983b) Visualization of anti-sperm plasma membrane IgG and $\mathrm{Fab}$ as a method for localization of sperm plasma membrane antigens. J. Histochem, Cytochem. 30, $1217-1227$.

Russell, L.D., Peterson, R.N., Hunt, W.P. \& Strack, L.E. (1984) Post testicular surface modifications and contributions of reproductive tract fluids to the surface polypeptide composition of boar spermatozoa. Biol. Reprod. 30, 959-978.

Russell, L.D., Montag, B., Hunt, W. \& Peterson, R.N. (1985) A qualitative and quantitative analysis of polypeptides released from epididymal and ejaculated sperm upon washing. Gamete Res. 11, 237-252.

Saxena, N.K., Russell, L.D., Saxena, N. \& Peterson, R.N. (1986a) Immunofluorescence antigen localization on boar sperm plasma membranes. Monoclonal antibodies reveal apparent new domains and apparent redistribution of surface antigens during maturation and at ejaculation. Anat. Rec. 214, 238-252.

Saxena, N.K., Saxena, N., Peterson, R.N., Henry, L., Hunt, W.P. \& Russell, L.D. (1986b) Increase in concentration of major sperm surface proteins during maturation in the epididymis. J. Cell Sci. 82, 295-308.

Tamblyn, T.M. (1980) Identification of actin in boar epididymal spermatozoa. Biol. Reprod. 22, 727-734.

Virtanen, I., Baadley, R.A., Paasivuo, R. \& Lehto, V.P. (1984) Distinct cytoskeletal domains revealed in sperm cells. J. Cell Biol. 99, 1083-1091. 